\title{
Oral L-serine supplementation reduces production of neurotoxic deoxysphingolipids in mice and humans with hereditary sensory autonomic neuropathy type 1
}

\author{
Kevin Garofalo, ${ }^{1}$ Anke Penno, ${ }^{2}$ Brian P. Schmidt, ${ }^{1}$ Ho-Joon Lee, ${ }^{3}$ Matthew P. Frosch, ${ }^{4}$ \\ Arnold von Eckardstein, ${ }^{2}$ Robert H. Brown, ${ }^{5}$ Thorsten Hornemann, ${ }^{2}$ and Florian S. Eichler ${ }^{1}$

\begin{abstract}
${ }^{1}$ MGH Neuroscience Center, Department of Neurology, Harvard Medical School, Boston, Massachusetts, USA. ${ }^{2}$ Institute for Clinical Chemistry, University Hospital Zurich, Zurich, Switzerland. ${ }^{3}$ Vincent Center for Reproductive Biology and ${ }^{4}$ C.S. Kubik Laboratory for Neuropathology, Department of Pathology, Massachusetts General Hospital, Harvard Medical School, Boston, Massachusetts, USA. ${ }^{5}$ Department of Neurology,
\end{abstract} \\ University of Massachusetts Medical School, Worcester, Massachusetts, USA.
}

\begin{abstract}
Hereditary sensory and autonomic neuropathy type 1 (HSAN1) causes sensory loss that predominantly affects the lower limbs, often preceded by hyperpathia and spontaneous shooting or lancinating pain. It is caused by several missense mutations in the genes encoding 2 of the 3 subunits of the enzyme serine palmitoyltransferase (SPT). The mutant forms of the enzyme show a shift from their canonical substrate L-serine to the alternative substrate L-alanine. This shift leads to increased formation of neurotoxic deoxysphingolipids (dSLs). Our initial analysis showed that in HEK cells transfected with SPTLC1 mutants, dSL generation was modulated in vitro in the presence of various amino acids. We therefore examined whether in vivo specific amino acid substrate supplementation influenced dSL levels and disease severity in HSAN1. In mice bearing a transgene expressing the C133W SPTLC1 mutant linked to HSAN1, a 10\% L-serine-enriched diet reduced dSL levels. L-serine supplementation also improved measures of motor and sensory performance as well as measures of male fertility. In contrast, a 10\% L-alanine-enriched diet increased dSL levels and led to severe peripheral neuropathy. In a pilot study with 14 HSAN1 patients, L-serine supplementation similarly reduced dSL levels. These observations support the hypothesis that an altered substrate selectivity of the mutant SPT is key to the pathophysiology of HSAN1 and raise the prospect of $\mathrm{L}$-serine supplementation as a first treatment option for this disorder.
\end{abstract}

\section{Introduction}

Hereditary sensory and autonomic neuropathy type 1 (HSAN1) is caused by several missense mutations in the SPTLC1 and SPTLC2 genes, which encode 2 of 3 subunits of the enzyme serine palmitoyltransferase (SPT) (1-4). No mutations in the third SPT subunit, SPTLC3, have yet been associated with HSAN1 (4). SPT catalyzes the first and rate-limiting step in the de novo synthesis of sphingolipids, namely the condensation of L-serine and palmitoyl-CoA (4, $5)$. The reaction product, 3-ketosphinganine, is quickly converted to sphinganine (SA), which is subsequently $\mathrm{N}$-acylated to dihydro-ceramide and finally converted to ceramide. Complex sphingolipids are generated by adding various head groups to the $\mathrm{C} 1$ hydroxyl group of ceramide.

To date, 5 SPTLC1 mutations, C133W, C133Y, V144D, S331F, and A352V, have been conclusively linked to HSAN1 (6-8). A common feature of all HSAN1 mutants is reduced in vitro activity of the enzyme, as measured by the incorporation of labeled L-serine (9). Besides this reduced reactivity with its canonical substrate L-serine, it appears that the mutant SPT has a promiscuously increased reactivity, with L-alanine and (at least in part) L-glycine as alternative substrates (10-12). This results in the formation of an atypical class of deoxysphingolipids (dSLs), which are composed either of deoxysphinganine (doxSA) when alanine is used by SPT

Authorship note: Florian S. Eichler and Thorsten Hornemann are co-senior authors. Conflict of interest: The authors have declared that no conflict of interest exists. Citation for this article: JClin Invest. 2011;121(12):4735-4745. doi:10.1172/JCI57549.
(Figure 1), or of deoxymethylsphinganine (doxmethSA) when glycine is used. Like regular sphingolipids, dSLs are also N-acetylated and finally converted to a deoxysphingosine (doxSO) backbone by the introduction of C4-5 double bond (12). However, because dSLs lack the $\mathrm{C} 1$ hydroxyl group of ceramides, they are therefore not converted to complex sphingolipids like phospho- or glycosphingolipids, nor degraded by the classical catabolic pathway, which requires the formation of the catabolic intermediate sphingosine-1-phosphate. dSLs were found to be highly elevated not only in cells expressing the mutant forms of SPT, but also in plasma of HSAN1 patients and in mice bearing a transgene expressing mutant SPTLC1 $(10,13)$. These observations led to the hypothesis that an altered substrate selectivity of the mutant SPT is the underlying key to the pathophysiology of HSAN1 $(10,13)$.

The clinical phenotype of HSAN1 is one of early dissociated sensory involvement affecting pain and temperature with preservation of vibration and joint position sense caused by a lengthdependent axonal neuropathy (6). There are frequent positive sensory symptoms of severe shooting or burning pain in the limbs and an early but transient period of hyperpathia in some patients. Because of the profound sensory impairment, many affected individuals develop neuropathic ulcers and Charcot joints, requiring amputation. There is prominent and often early motor involvement in most patients. Autopsy reports from HSAN1 patients show pronounced degeneration of dorsal root ganglion cells with depletion of myelinated axons in peripheral nerve $(6,14-16)$. Loss of unmyelinated axons is present, but reported to be less severe. 


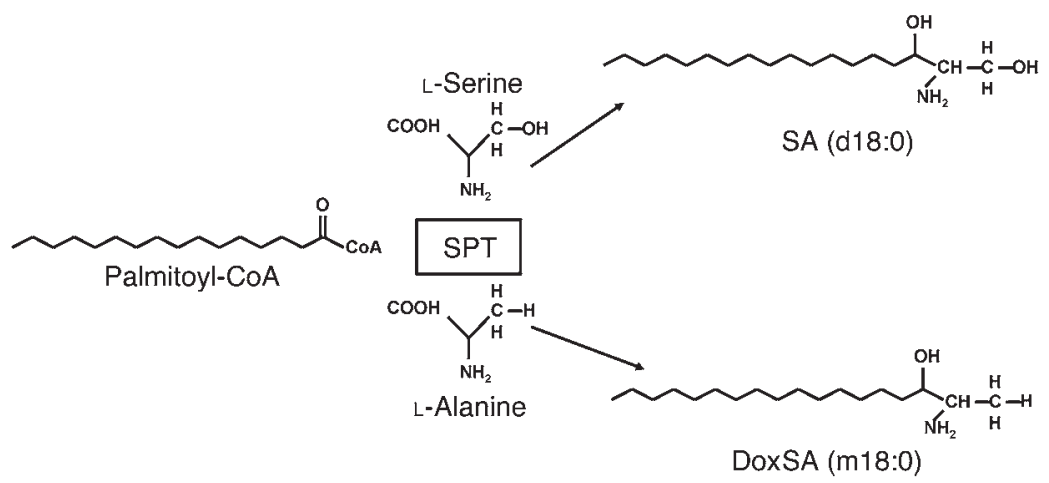

\section{Figure 1}

Sphingolipid de novo synthesis is initiated by the condensation of an activated fatty acid (normally palmitoyl-CoA) and L-serine to form SA. This reaction is catalyzed by SPT. In HSAN1, the SPT shows a shift in substrate specificity to alanine, which results in the formation of an atypical class of dSLs. For SA and doxSA (1-deoxysphinganine), the number of hydroxyl groups is indicated by $\mathrm{m}$ (mono-) and $\mathrm{d}$ (di-), followed by the number of carbons; the second number indicates the double bonds.
Transgenic mice with the C133W mutation closely mimic the pattern of disease progression in humans. By 10 months of age, transgenic mice develop hyperpathia and motor deficits, peripheral myelin thinning, loss of visceral innervation, and indications of neuronal stress within the dorsal root ganglia (17). At the age of 12 months, mice are no longer hyperpathic, and by 14 months, they manifest a small fiber sensory neuropathy and deficits in sensory and motor function similar to those of HSAN1 patients (13). Unlike humans, the C133W transgenic mice display infertility and have decreased sperm count, sperm motility, and testes weight, features that can be reversed by forced overexpression of the WT gene in double-transgenic mice $(10,13)$.

Based on the mechanism of deoxysphingoid base generation, we hypothesized that L-serine supplementation would prove to be beneficial in mice and humans with HSAN1. L-serine is essential for neuronal survival. Hereditary conditions of decreased L-serine synthesis cause a severe neurological phenotype (18-20). Biochemical analysis has shown that L-serine is synthesized from glucose and released by astrocytes, but not by neurons. The peripheral nerve lacks the abundance of astrocytes found in the CNS, which is why the dorsal root ganglion may be particularly vulnerable in HSAN1. In contrast, we hypothesized that L-alanine would have a detrimental effect for transgenic mice harboring the HSAN1 mutation within the SPTLC1 gene. Our comparison of these closely related yet divergent effects in HSAN1 and their effect on the nervous system shed light on the role of sphingolipids in neuronal survival and health.

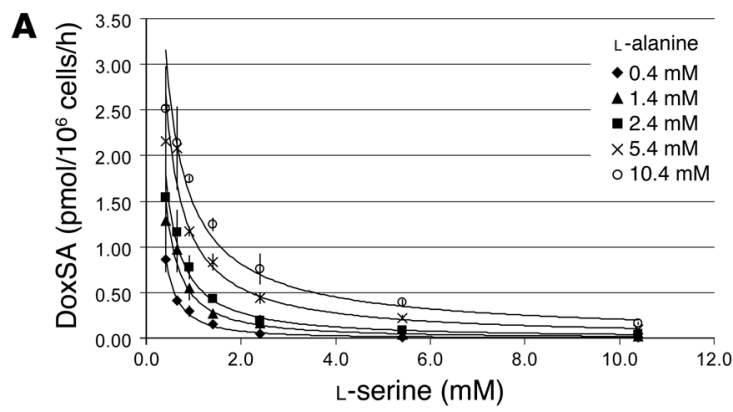

\section{Results}

\section{L-serine suppresses doxSA generation in HEK293 cells}

Increasing L-alanine concentration in the medium increased doxSA formation in cultured SPTLC1 WT cells, whereas increasing L-serine medium concentrations substantially suppressed endogenous doxSA production, even in the presence of elevated alanine levels (Figure 2A). This demonstrates competitive inhibition between L-serine and L-alanine. SA production, in contrast, was greatly increased in the presence of L-serine and not markedly suppressed in the presence of L-alanine.

DoxSA production at a constant 2-mM L-alanine background was clearly higher for the HSAN1 mutant-expressing versus WT cells (Figure 2B). Similar to the WT SPTLC1-expressing cells, we observed suppression of doxSA production in HSAN1 mutantexpressing cells with increasing L-serine medium concentrations (Figure 2B), which indicates that the noxious dSL production in HSAN1 could in principle be overcome by increased systemic availability of L-serine.

\section{Supplementation increases respective amino acid levels in $\mathrm{C133W}$ mice}

We confirmed that dietary supplementation resulted in increased plasma levels of the respective amino acids (Figure 3A). Amino acid levels in sciatic nerve and liver showed a similar pattern (Figure 3, B and C). In both tissues, the serine/alanine ratio was higher in L-serine- and lower in L-alanine-supplemented mice compared with untreated $\mathrm{C} 133 \mathrm{~W}$ mice.

B

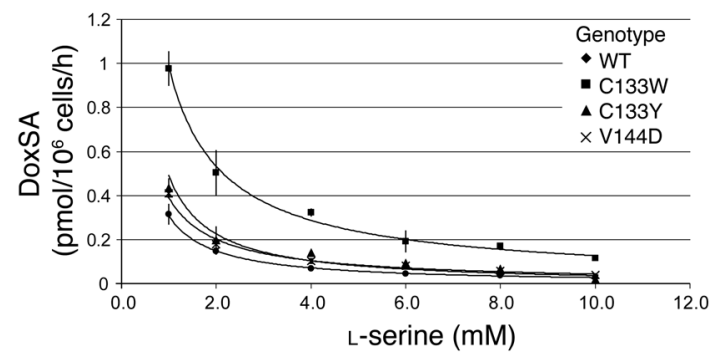

\section{Figure 2}

Kinetic characterization of WT and mutant SPT. (A) Suppression of doxSA production in SPTLC1 WT HEK cells by increasing L-serine medium concentrations in the background of varying L-alanine concentrations. (B) Suppression of doxSA generation in SPTLC1 WT and HSAN1 mutantexpressing HEK293 cells at increasing L-serine medium concentrations (constant background of $2 \mathrm{mM} \mathrm{L-alanine).}$ 

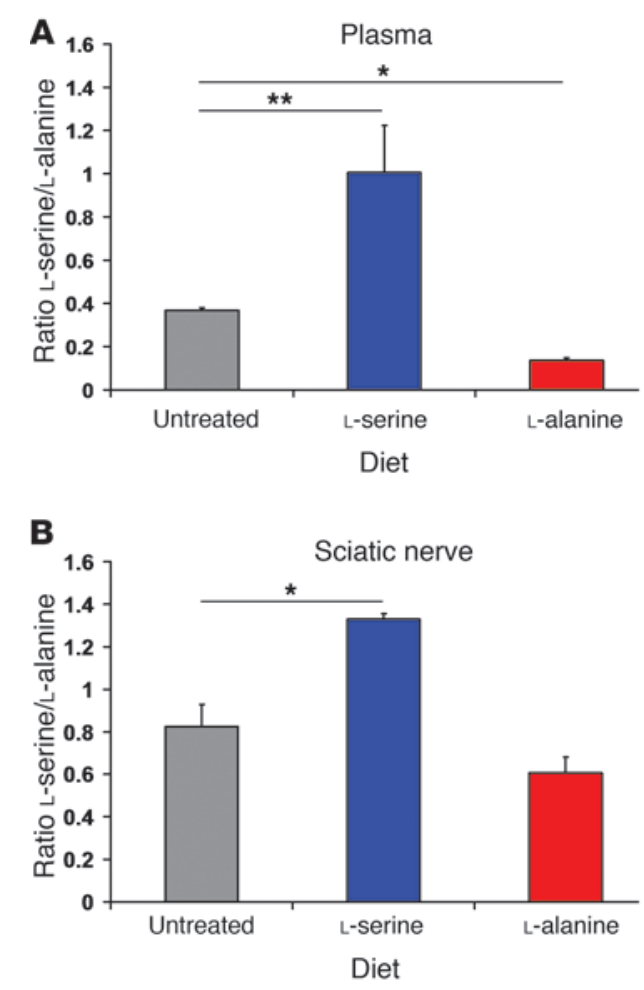

\section{Supplementation in C133W mice alters dSL levels}

Sphingoid bases in plasma are usually $\mathrm{N}$-acetylated. To directly analyze the sphingoid base composition of these metabolites, we subjected the extracted lipids to sequential acid and base hydrolysis to release the $\mathrm{N}$-acyl chain. The resulting free deoxysphingoid base backbones were analyzed by liquid chromatography-mass spectrometry. In support of our hypothesis, C133W mutant mice on a $10 \%$ L-serine-enriched diet showed a roughly 5 -fold decrease of doxSA-based sphingolipids $(P<0.001)$ and an approximately 10 -fold decrease of doxSO-based sphingolipids $(P<0.001)$, reaching levels comparable to those of WT mice within 2-4 days (Figure 4, B and D). In contrast, C133W mice on a 10\% L-alanine-enriched diet showed elevated, highly oscillating doxSA and doxSO plasma levels that, on average, increased over time $(P<0.05$ and $P<0.001$, respectively).

In the same mice, total levels of sphingosine- (SO-) and SAbased sphingolipids over the time period did not differ significantly between the 2 groups (L-serine versus L-alanine supplementation). However, we did note a significant increase over time for both SA- and SO-based sphingolipids in L-serine-fed, but not L-alanine-fed, mice $(P<0.01)$. On average, plasma SO showed a slow but consistent increase, whereas plasma SA increased until day 9 and then returned to approximately baseline levels (Figure 4, A and C).

Once on the L-serine diet, transgenic mice were protected from neurodegeneration on measures of both mechanical sensitivity (von Frey) and motor performance (rotarod). Mice on the $10 \%$ L-serine diet retained their neurological function up to 15 months of age, unlike the untreated mice, who by that age had developed a neuropathy. In contrast, the L-alanine-supplemented mice had developed a severe peripheral neuropathy by 5 months of age. Details of their performance are presented below.

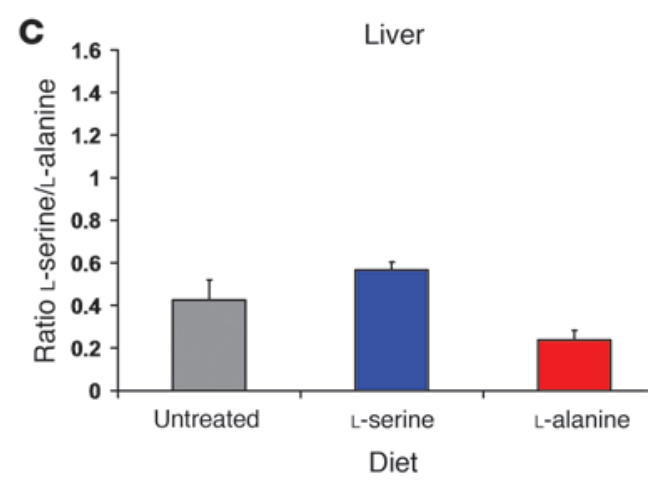

Figure 3

Effects of supplementation on L-serine/L-alanine concentrations. C133W transgenic mice were fed diets with 10\% L-serine, 10\% L-alanine, or were left untreated. (A) Plasma levels after 3 days on diet demonstrated a significant increase in the ratio of L-serine to L-alanine in plasma of L-serine-fed mice relative to the untreated group. In contrast, mice fed L-alanine had a decrease in amino acid ratio. (B) Sciatic nerve showed a similar change in the L-serine/L-alanine ratio after 9-12 months of supplementation. (C) Liver did not show significant differences between treatment groups. ${ }^{\star} P<0.05$; ${ }^{\star \star} P<0.01$. Error bars represent SEM.

\section{Effects of short-term, 2-month supplementation on neurological measures}

Motor performance. Young C133W mice on L-alanine for 2 months (Figure 5A) exhibited a trend toward slightly worse performance on rotarod by 5 months of age (Figure 5B). This was a mild exacerbation of the mutant condition that did not show motor abnormalities until 12 months of age. Older C133W mice that received 2-month shortterm treatment with L-serine beginning at 13 months of age showed no benefit on rotarod (Figure 5D). Neither L-serine nor L-alanine diet significantly affected performance of WT animals (data not shown).

Mechanical and thermal sensitivity. Mechanical sensitivity worsened in 5-month-old C133W mice after 2 months of L-alanine treatment $(P<0.05$; Figure 5C). The threshold of mechanical sensitivity increased 3-fold compared with both presymptomatic C133W and WT control mice, indicative of early exacerbation of the phenotype. Short-term treatment of L-serine was tested in mildly symptomatic (146\% of WT threshold, consistent with decreased sensitivity) older C133W mice beginning at 13 months of age. Despite already being symptomatic, these older C133W mice displayed a trend toward rescuing mechanical sensitivity after 2 months of L-serine treatment (95\% of WT; Figure 5E).

\section{Effects of long-term, 10-month supplementation on neurological measures}

In contrast to short-term-treated mice, which primarily showed improvement in mechanical sensitivity, long-term L-serine treatment for 10 months improved rotarod performance of C133W mice compared with the untreated condition $(P<0.05$; Figure 5F). Long-term L-serine treatment also resulted in a trend of improved mechanical sensitivity compared with the untreated C133W condition (146\% of WT threshold vs. 96\%; Figure 5G). No significant differences were seen on hotplate testing after short- or long-term treatment. 
A

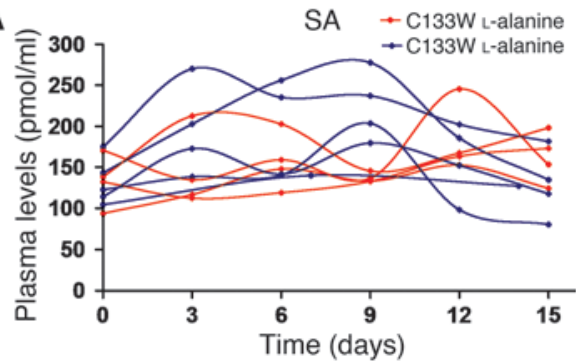

B

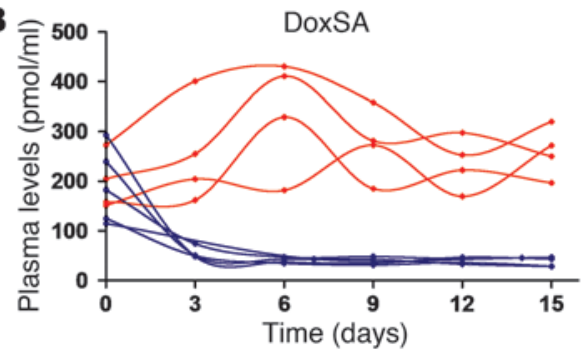

C
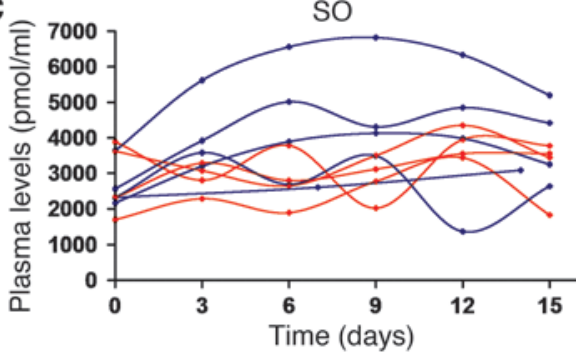

D

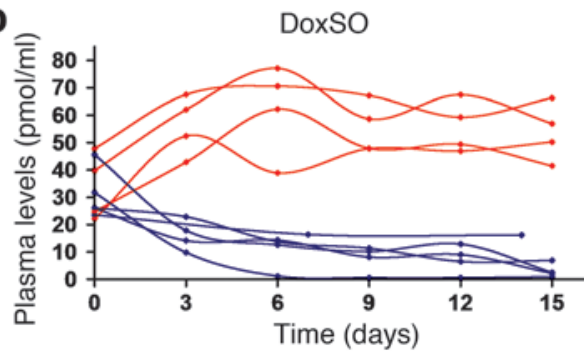

Figure 4

Effects of supplementation on plasma dSL levels in C133W transgenic mice. C133W transgenic mice were fed either $10 \%$ L-serine-supplemented or $10 \%$ L-alanine-supplemented diet. Plasma samples were drawn every third day. Lipids were extracted and subjected to acid, and base hydrolysis and free sphingoid bases were quantified by liquid chromatography-mass spectrometry. (A) SA levels did not differ as a consequence of supplementation. (B) L-serine diet induced a significant reduction in doxSA plasma levels within the first 3 days. L-alanine caused an initial increase in doxSA levels and subsequent fluctuations. (C) Dietary supplementation did not significantly affect SO plasma levels. (D) DoxSO levels decreased after L-serine supplementation, while increasing in response to L-alanine.

After 12 months of treatment with L-serine, total doxSA levels were by trend lower in tissue (liver, sciatic nerve, brain, and spine) of C133W transgenic compared with untreated mice (Figure $6 \mathrm{~B})$. In contrast, L-alanine-treated mice showed generally higher doxSA levels than the untreated or L-serine-treated groups. This difference was most pronounced in liver tissue, in which doxSA levels increased up to 3 -fold compared with $\mathrm{C} 133 \mathrm{~W}$ and WT mice $(P<0.01$ and $P<0.05$, respectively). A similar, though nonsignificant, trend was also found in sciatic nerve, brain, and spinal tissue. Interestingly, with L-alanine supplementation, a few C133W mice developed skin ulcers, a feature commonly described in HSAN1 patients.

As above, we observed dramatically increased doxSO levels in sciatic nerve of the C133W mice (Figure 6D). No doxSO was found in any tissue of WT mice. Interestingly, doxSO levels were not significantly altered in response to the diet. DoxSO was also found in the livers of C133W mice, but not in brains or spinal cords. In liver, doxSO levels were significantly elevated in the L-alanine-fed compared with the unsupplemented or L-serinesupplemented C133W mice.

The influence of $\mathrm{L}$-serine supplementation was also reflected in altered SA levels (Figure 6A). SA levels were especially elevated in liver tissue compared with those of control or L-alanine-fed mice $(P<0.05)$. A trend toward higher SA levels was also observed in sciatic nerve, but seemed to be reversed in brain and spine. Moreover, $\mathrm{SO}$ was marginally higher in liver and sciatic nerve of $\mathrm{L}$-serine-fed mice than in those receiving L-alanine $(P<0.06$; Figure $6 \mathrm{C})$. Interestingly, total SO levels were about 50\% lower in C133W than WT mouse sciatic nerve, despite the fact that levels in plasma or other tissue (liver, brain, or spine) were not altered. This indicates that the sciatic nerve seems to be less capable than other tissues of compensating for the reduction in SPT activity.

\section{Effect of treatment duration on behavioral performance}

We also compared the behavioral performance of the animals with respect to the duration of L-serine treatment (2 versus 10 months; Figure 5, $\mathrm{D}-\mathrm{G})$. On the rotarod, mutant mice treated for 10 months scored significantly better than those treated for 2 months $(P<0.01)$. Long-term effects on mechanical and thermal sensitivity showed the same trend, but the differences were not significant.

\section{Effect of supplementation on peripheral nerve}

On L-serine, C133W mice showed no difference in axon density of myelinated sciatic nerves, but the number of unmyelinated fibers was 2 -fold higher $(P=0.02)$ than that in untreated C133W mice of the same age (13). Both myelinated and unmyelinated axons of C133W mice fed L-serine showed a trend toward a larger mean diameter compared with the untreated condition (myelinated, 3.97 vs. $2.64 \mu \mathrm{m}$; unmyelinated, 0.63 vs. $0.54 \mu \mathrm{m}$; Figure 7). C133W mice fed L-alanine, despite their younger age (9 months), had smaller mean myelinated and unmyelinated axon diameter (3.49 $\mu \mathrm{m}$ and $0.54 \mu \mathrm{m}$, respectively) and abnormal Schwann cells, consistent with degeneration of the distal sciatic nerve.

\section{Effect of supplementation on sperm count, sperm motility, and testis weight}

Older mice expressing the $\mathrm{C} 133 \mathrm{~W}$ mutation are known to have a decreased sperm count (13). After 4 months of L-serine supplementation (Figure 8A), the sperm count of C133W mutant mice improved significantly compared with untreated $\mathrm{C} 133 \mathrm{~W}$ animals $(P<0.05$; Figure $8 \mathrm{~F})$. Testis weight and sperm motility were not significantly different between groups; however, L-serine-treated C133W mice had by trend higher sperm motility and testis weight than did untreated C133W mice (Figure 8, E and G). Untreated young (8 months) C133W mice did not differ significantly from age-matched WT controls, although all 3 measures returned means less than WT. Additionally, C133W mice fed with L-alanine for 5 months had levels of all 3 measurements similar to those of age-matched WT controls and were not significantly different from C133W control mice (Figure 8, B-D).

\section{Pilot study in humans with the C133Y mutation}

The above results showed that oral L-serine supplementation markedly decreased plasma dSL levels in association with significant neurological improvement in the HSAN1 mouse model. We therefore sought to confirm these findings in human HSAN1 


\section{A}

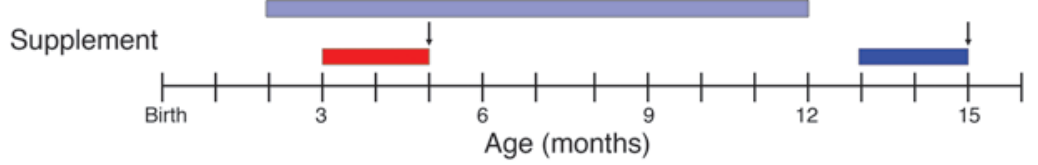

B

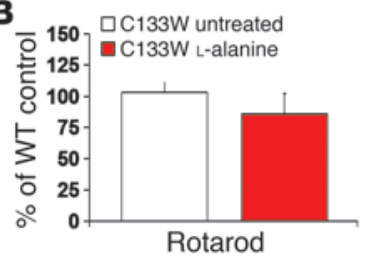

C

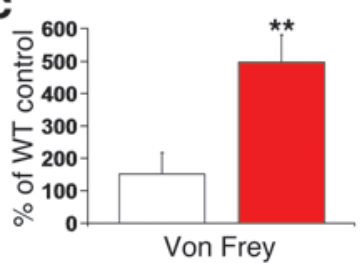

D

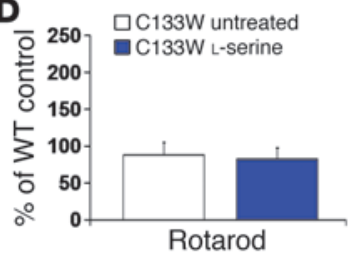

E

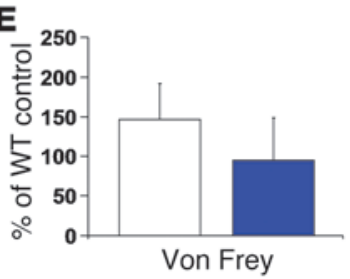

$\mathbf{F}$

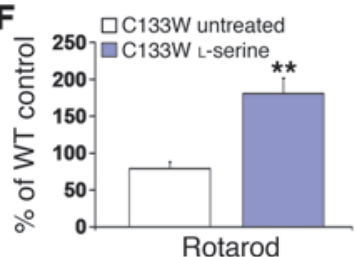

G

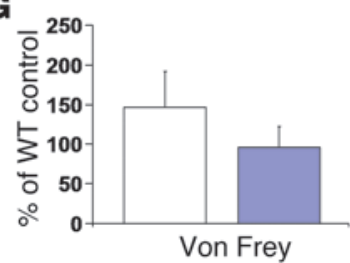

Figure 5

Effects of supplementation on behavior in C133W transgenic mice. (A) Experimental design. Red bar, L-alanine supplementation; blue bars, L-serine supplementation; arrows, time of sensory testing. (B) In L-alanine-supplemented mice, a trend toward worse motor performance was seen on rotarod. (C) Mechanical sensitivity was significantly worse compared with untreated age-matched C133W transgenic and WT mice. (D) In contrast, L-serine had no effect on the motor performance of 15-month-old transgenic mice, but (E) a beneficial effect on mechanical sensitivity was seen. (F) Long-term L-serine treatment improved motor performance compared with untreated age-matched C133W transgenic and WT mice. (G) Mechanical sensitivity showed a trend toward improvement relative to untreated C133W transgenic mice. Data (mean \pm SEM) show percentage of age-matched, control diet-fed WT value. ${ }^{\star \star} P<0.05$ vs. untreated C133W transgenic mice.

patients. In a pilot study, 14 HSAN1 patients were treated with either low- or high-dose L-serine $(200$ or $400 \mathrm{mg} / \mathrm{kg} / \mathrm{d}$, respectively; Figure 9A). All patients carried the C133Y SPTLC1 mutant. Clinical data on the patients are provided in Supplemental Table 1 (supplemental material available online with this article; doi:10.1172/JCI57549DS1).

We observed dramatic lowering of plasma dSL levels in both groups (Figure 9). Patients on the low-dose regimen showed a roughly 2 -fold decrease of dSL levels within a month, while patients on the high-dose regimen had closer to a 4-fold decrease in the same time period. Full analysis of the 10 -week period showed that a nadir in both regimens occurred at 6 weeks, with persistent reduction thereafter, and that both treatments caused a highly significant reduction in dSLs as a function of time $(P<0.001)$. Additionally, we noted a trend of dose dependence across both dSL levels, although these findings were not significant within our limited sample size. Interestingly, SA and SO increased significantly in both high- and low-dose regimen patients $(P<0.001)$ - again in a manner suggestive of dose dependence (data not shown). After completion of the 10-week trial, dSL levels began to rise again during the 2 -week washout period. Improvement in neurological symptoms were not examined and also not expect-

\section{Figure 6}

Effect of 9-12 months' dietary supplementation on tissue dSL levels in C133W transgenic mice. (A) L-serine caused a significant increase of SA in liver. (B) Compared with untreated controls, doxSA levels decreased slightly with L-serine treatment and increased with L-alanine treatment in C133W transgenic mice. (C) No significant trend was noted for SO tissue levels. (D) DoxSO was found in liver and sciatic nerve, but showed no apparent dependence upon dietary supplementation. (Because doxSO was not detected in tissue of WT mice on a control diet, mean values are expressed in pmol per $\mu \mathrm{g}$ protein). Error bars represent SEM. ${ }^{* *} P<0.05$.
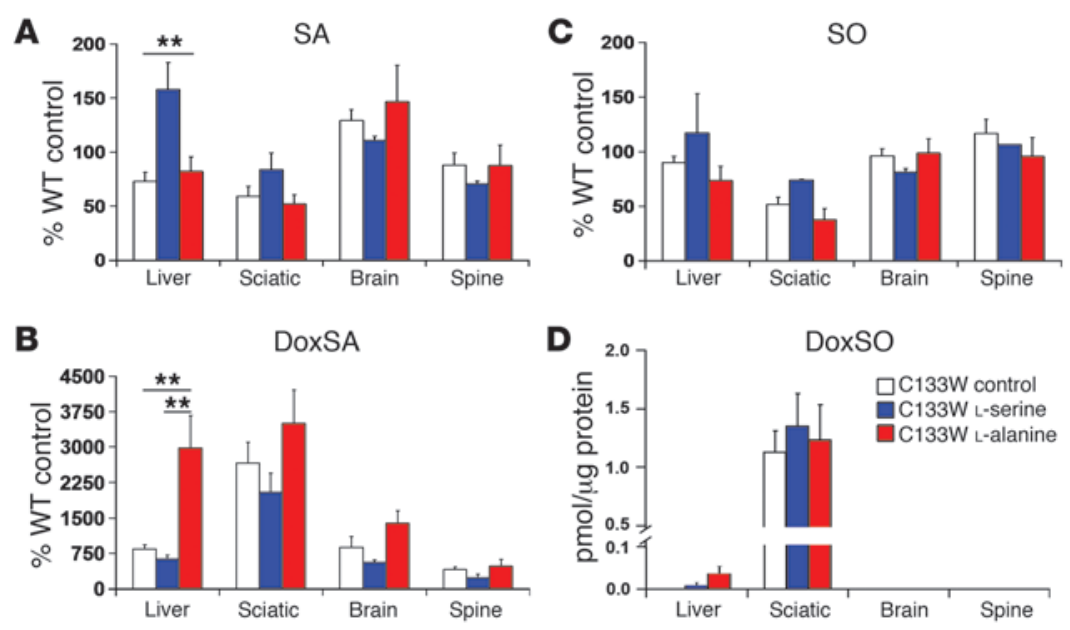

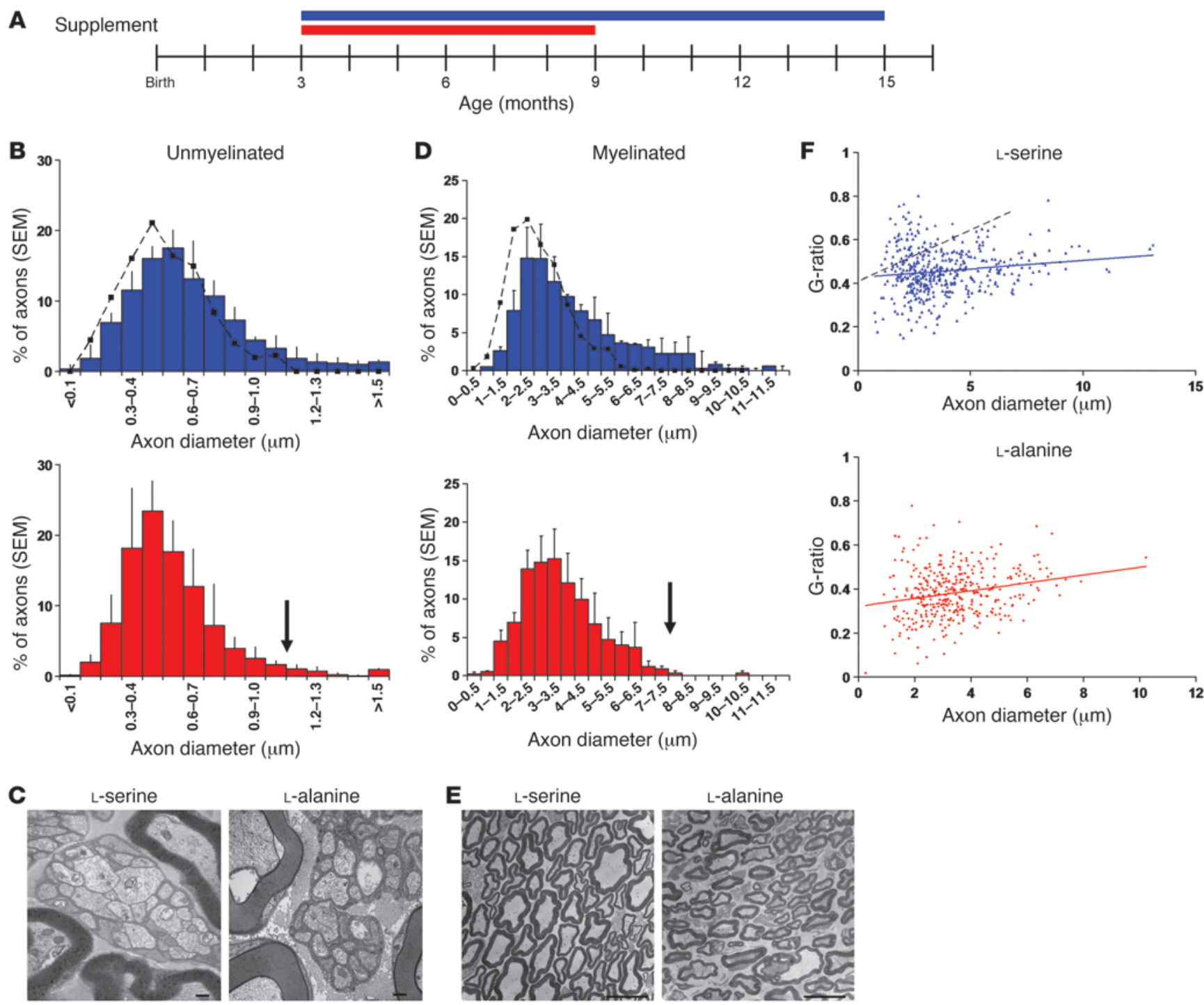

Figure 7

Effects of supplementation on sciatic nerve morphology. C133W transgenic mice were fed diets with $10 \%$ L-serine or $10 \%$ L-alanine. (A) Experimental design. Red bar, L-alanine supplementation; blue bar, L-serine supplementation; end of bar denotes time of sacrifice. (B) Quantification of unmyelinated axons revealed rescue of large-caliber axons in young L-serine-treated mice (blue) relative to age-matched untreated C133W transgenic animals (dotted black line; data from ref. 13) and young L-alanine-treated mice (red). Arrow indicates relative decrease in large-caliber axons. (C) EM images of unmyelinated axons from L-serine- and L-alanine-fed mice. Scale bars: $500 \mathrm{~nm}$. (D) Myelinated axons in L-serine-treated animals demonstrated a similar trend of preservation of large axons. Dotted black line represent age-matched untreated $\mathrm{C} 133 \mathrm{~W}$ transgenic animals (data from ref. 13). Arrow indicates relative decrease in large-caliber axons. (E) EM images of myelinated axons from L-serine- and L-alanine-fed mice. Scale bars: $10 \mu \mathrm{m}$. (F) Change in myelination patterns after supplementation, assessed by g ratios. Dotted black line represents old untreated C133W transgenic animals (data from ref. 13). Error bars represent SEM.

ed within the time frame of this study. However, some patients reported an increase in sensation (hand tingling, increased menstrual cramps) and great improvements of skin robustness as well as faster nail and hair growth.

\section{Discussion}

Under normal circumstances, SPT catalyzes the condensation of L-serine with palmitoyl-CoA to form SA. After the discovery in 2001 that several point mutations in the SPTLC1 subunit of SPT were linked to the dominantly inherited neuropathy, HSAN1, it was thought that reduced SPT activity (i.e., haploinsufficiency) was the mechanism responsible for pathology $(21,22)$. Indeed, expression of HSAN1 mutants in yeast and cell culture dominantly inactivated SPT activity, and HSAN1 lymphoblasts also showed 50\% reduced activity in vitro (9). However, the reduced SPT activity did not result in a reduction of total plasma sphingoid base levels in the patients $(9,17)$. Furthermore, heterozygous SPTLC1 knockout mice $(13,23)$ showed significantly reduced SPT activity, but did not develop neurological impairments (homozygous SPTLC1 knockout mice are embryonic lethal). We recently reported that mutations in HSAN1 
A

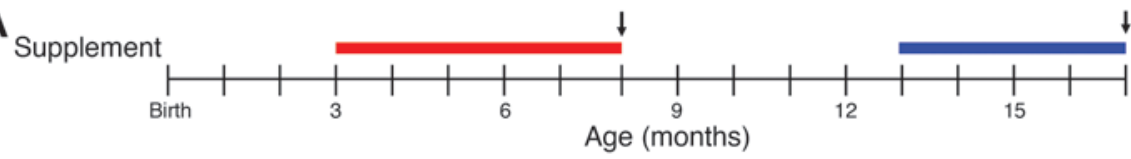

B

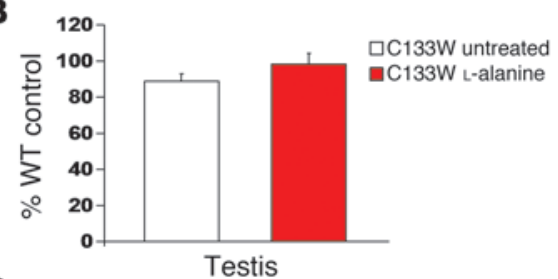

C

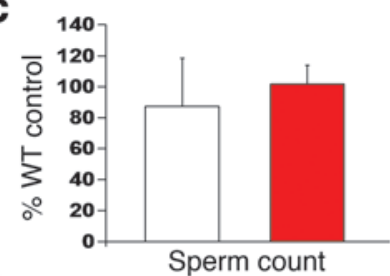

D

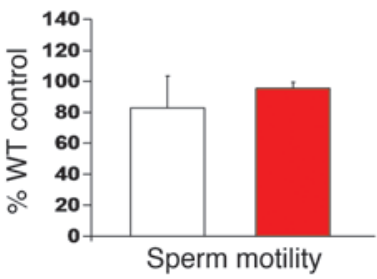

E
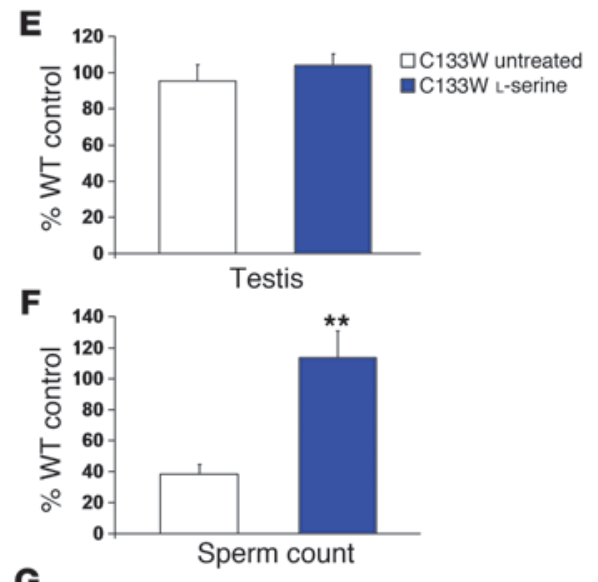

G

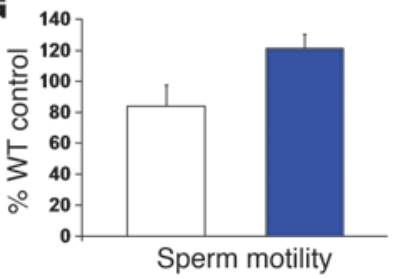

\section{Figure 8}

Effects of supplementation on the reproductive system of $\mathrm{C} 133 \mathrm{~W}$ transgenic mice. (A) Experimental design. Red bar, L-alanine supplementation; blue bar, L-serine supplementation; arrows, time of reproductive analysis. (B-D) Young C133W transgenic mice fed L-alaninesupplemented diet did not differ from C133W transgenic or WT control animals on measures of testes weight (B), sperm count (C), or sperm motility (D). (E-G) L-serine-treated C133W transgenic mice were found to have increased sperm count $(\mathbf{F})$, but no differences in testes weight $(\mathbf{E})$ or sperm motility $(\mathbf{G})$. Data (mean \pm SEM) show percentage of age-matched, control diet-fed WT value. ${ }^{\star \star} P<0.05$. lead to increased production of previously unrecognized atypical dSLs that cannot be degraded by the canonical sphingolipid catabolism (10-13). An alternative explanation for the presumed haploinsufficiency is that accumulation of these lipids may drive HSAN1 pathology via a toxic gain-of-function mechanism $(10,13)$. Our present report lends further support to this hypothesis.

In this study, we investigated the effect of HSAN1 point mutations on SPT substrate affinity and dSL generation in 2 model environments (in HEK cells transfected with the C133W, C133Y, and V144D mutation and in C133W transgenic mice) and in human HSAN1 patients harboring the C133Y mutation. In all 3 instances, L-serine was able to inhibit dSL generation. Most importantly, with L-serine supplementation, C133W transgenic mice showed improved neurological performance and male fertility. As dSLs show neurotoxic features in vitro, effective substrate supplementation and suppression of dSL formation may represent a potential treatment for HSAN1.

First, we characterized WT and HSAN1 mutant SPT activity in HEK cells. By increasing L-serine concentrations in culture media, we were able to modulate doxSA generation in the presence of varying L-alanine concentrations. In contrast, L-serine supplementation suppressed doxSA generation for the C133W, C133Y, and V144D mutants in transfected HEK cells. A previous report indicated that the HSAN1 mutations primarily change the enzymatic activity $\left(V_{\max }\right)$ with L-serine and L-alanine, not the affinity $\left(K_{m}\right)$ for these substrates (11). Therefore, we concluded that increasing the L-serine/L-alanine ratio results in a preferential formation of SA over doxSA in the cell.

This prompted us to validate the effects of dietary amino acid supplementation on dSL levels and neurological function in C133W transgenic mice. With the $10 \%$ L-serine-enriched diet, we observed a rapid reduction in plasma dSL levels into the normal range within 3-4 days. Subsequent long-term L-serine-fed mice retained their neurological functions up to 15 months of age, an age at which untreated C133W mice invariably developed a severe neuropathy. Even short-term supplementation in older transgenic mice brought about a trend toward improved mechanical sensitivity, which - while not as significant as the improvement in younger mice - suggested that even in advanced disease, there may still be a role for supplementation. In stark contrast, L-alanine feeding raised dSL levels and significantly worsened the disease. The threshold of mechanical sensitivity increased 5-fold in L-alanine-treated compared with untreated WT animals, indicative of a severe loss of sensation, like human patients who unknowingly injure themselves due to their sensory deficits (6). Furthermore, some of the L-alanine-supplemented $\mathrm{C} 133 \mathrm{~W}$ mice also developed skin ulcers, which was not observed in L-serine-fed C133W mice.

Prior studies on overexpression of WT SPTLC1 corroborate our data supporting the link between dSL accumulation and neurodegeneration. While C133W transgenic mice develop sensory and motor impairments after a year of age, co-overexpression of the WT and the mutant SPTLC1 subunit in double transgenic mice prevents neuronal loss and the deficits in sensory and motor function (13). In concordance with this, elevated levels of dSL were found in plasma of C133W transgenic mice, while double transgenic mice showed significantly lower dSL levels, in the intermediate range between WT and C133W transgenic mice (13).

Our mouse model also allowed us to analyze the accumulation of dSL within specific tissue compartments. Previously, we showed that dSL levels in C133W mutant mice are markedly elevated in affected tissue, such as sciatic nerve and testes, compared with WT mice, in contrast to unaffected compartments of the nervous system, such as brain and spinal cord, where there is little or no accumulation of dSL (13). 

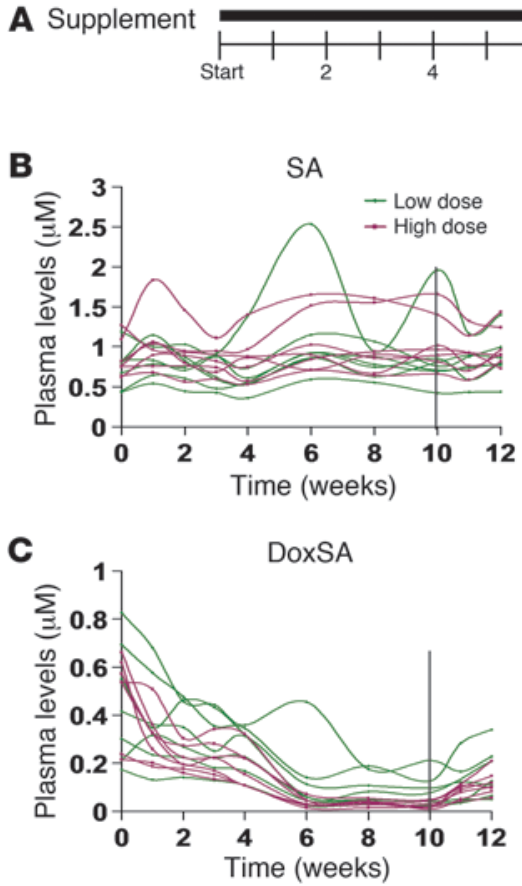

\section{Figure 9}

Effects of L-serine supplementation on human HSAN1 patients. (A) Design of the pilot study. Solid line, L-serine supplementation period; gray line, washout period. Plasma levels of SA (B) and $\mathrm{SO}(\mathrm{D})$ increased substantially during L-serine treatment. In contrast, doxSA (C) and doxSO (E) decreased in HSAN1 patients, reached a nadir within 4-6 weeks, and showed greater suppression at the higher dose. During the washout phase, dSL levels began to rise. Vertical lines at 10 weeks denote the beginning of washout.
Our current studies showed that, by trend, doxSA levels were lower in the sciatic nerves of L-serine-fed and higher in L-alaninefed mice, but total doxSO levels were not different. Liver tissue, in contrast, showed a significant increase in dSL levels with L-alanine supplementation, but only a slight reduction with L-serine. This was in contrast to plasma dSL levels, which dramatically decreased upon L-serine treatment. However, the L-serine-treated mice showed clear improvements on motor and sensory testing. The apparent discrepancy between major functional changes and minor alterations in dSL levels might be explained by the fact that the lipids were analyzed in the entire nerve section, encompassing neurons, glia, and connective tissue, rather than in individual compartments. Small or local changes in dSL levels especially in functionally important microcompartments of the neurons - might therefore be blurred and poorly accounted for. The catabolism of dSLs is currently not known. Our data indicate that dSLs can be degraded in certain tissues, possibly by the action of a yet-unknown catabolic enzyme. A possible reason for the relatively selective accumulation of $\mathrm{dSL}$ in peripheral nerve tissue might be the absence of such an enzyme in neurons.

Neurotoxicity has been demonstrated for doxSA, but it is currently not known for other dSLs. DoxSA and associated deoxydihydroceramides are the most likely culprits for inducing toxicity in neurons, whereas doxSO and deoxyceramides are considered to be the metabolically inert end product of dSL metabolism and expected to accumulate in nerve and glia. Previous studies have shown an impaired cytoskeleton dynamic in neurons that were cultured in the presence of doxSA (10). There are indications from earlier works that doxSA exerts inhibitory actions on ceramide synthases and possibly also sphingosine kinase (24). An investigational marine anti-cancer drug, ES285 , which is chemically identical to doxSA, reduces actin stress fiber formation and disrupts rho-GTPase signaling in response to elevated doxSA levels (25). Moreover, in various cancer cell lines, doxSA appears to be cytotoxic (26), although increased cell death is not known to occur in either mice or humans carrying the HSAN1 mutations. Recently, it was also shown that GADD153, a marker for endoplasmic reticulum stress, is significantly elevated in yeast cells expressing mutant SPT heterotrimers (11). It is therefore possible that ER stress contributes to the pathomechanism of HSAN1. However, further functional studies are necessary to unravel the exact pathways underlying the neurotoxic effects of dSLs.

Unlike humans with HSAN1, the C133W mutant transgenic mice displayed poor fertility and reduced sperm count. Supplementation with L-serine resulted in a 3 -fold increase in sperm count and a trend toward increased sperm motility. Improved fertility was previously also seen with SPTLC1-overexpressing mice (13). We therefore speculate that L-serine supplementation may reduce the toxic effects of doxSA on testes. Apparently, for proper spermatogenesis, mice require the formation of specific testicular glycosphingolipids that contain polyunsaturated very long chain fatty acid residues in their ceramide moieties (27). Increased SA generation in L-serine-supplemented mice, on the other hand, may promote sperm production and motility $(27,28)$.

Having found sufficient evidence for a beneficial effect of L-serine in mice, we embarked on a human pilot study. Whereas our mice were created with the C133W mutation, the humans we included in our trial had the C133Y mutation. Nonetheless, we found analogous results with both mutations. Although the human L-serine dose was approximately 38 and 18 times less per kilogram body weight compared with mice on supplementary diet, the treatment resulted in a slower - but still significant - reduction of plasma dSL levels in both groups. After 6 weeks of L-serine treatment, the dSL levels of all but 1 patient were in the 95 th percentile of control individuals. Within this short pilot study, neurological function was not systematically assessed, but some patients reported a subjective increase in sensation, including enhanced menstrual cramps and a tingling feeling in the hands. Another volunteer described great improvement in 
skin robustness as well as in nail and hair growth. This is not surprising, as the importance of sphingolipids in the skin is well recognized, and HSAN1 is often accompanied by very delicate skin and ulcerations (6).

Although L-serine has previously been used to treat inherited metabolic disorders of L-serine biosynthesis $(19,20)$, to our knowledge, it has never been used for a disorder that has normal L-serine synthesis but dysfunctional use, nor has it been used as a neuroprotective agent. L-serine is thought to be essential for neuronal survival (18), and neurons may be especially susceptible to the HSAN1 mutation, if they have an unusually low serine/alanine ratio.

The toxic effects of dSLs on neurons may not be limited to HSAN1. Recently, Hornemann and colleagues demonstrated that dSL levels are elevated in diabetic patients (29), which suggests that dSLs may also contribute to the progression of diabetic sensory neuropathy. The peripheral neuropathy in advanced diabetes has a similar late onset (30). Slow progression, initial involvement of the distal extremities, and skin ulcers are features common to both HSAN1 and the neuropathy in diabetes.

A limitation of this human pilot study is that it was not designed to assess neurological outcome, but rather to evaluate biochemical effects. Therefore, despite signals that L-serine supplementation may be beneficial, we cannot yet draw conclusions about efficacy in humans. Nonetheless, given the efficacy of L-serine on reducing dSL levels in HEK cell lines, murine models, and humans with HSAN1, it is plausible and indeed likely that L-serine supplementation will be of value for the treatment of HSAN1. Therefore, a larger placebo-controlled trial is needed to prove whether longterm L-serine is safe and could prevent, arrest, or even reverse neurological impairment in HSAN1 patients.

\section{Methods}

\section{Cell culture}

HEK293 cells were obtained from the ATCC and cultured in Dulbecco modified Eagle medium (Sigma-Aldrich) with $10 \%$ fetal calf serum (Fisher Scientific FSA15-043) and penicillin/streptomycin (100 U/ml and $0.1 \mathrm{mg} / \mathrm{ml}$, respectively; Sigma-Aldrich). Transfection was done using Lipofectamine (Invitrogen). Stable cell lines were grown under the selection of $400 \mu \mathrm{g} / \mathrm{ml}$ Geneticin (Gibco, Invitrogen). Expression of SPTLC1 was confirmed by immunoblotting using anti-V5 and anti-His tag antibodies. For experimental procedures, media were changed and replaced with fresh media supplemented with varying concentrations of L-alanine, L-serine, and combinations thereof (0.4-10.4 mM). Cellular SPT activity and de novo synthesized sphingoid base metabolites were analyzed as described previously (10).

\section{Transgenic mice}

Transgene construction and generation of transgenic mice were carried out according to methods previously described (17). The final transgene construct consisted of the chicken $\beta$-actin promoter with cytomegalovirus immediate early gene-enhancer elements, followed by the SPTLC1 cDNA with $\mathrm{HA}$, and the rabbit $\beta$-globin polyadenylation signal. Mice were generated with standard techniques in the BL6/C57 background.

\section{Amino acid supplementation}

To test the hypothesis that amino acid supplementation alters disease progression, animals were placed on a rodent diet (AIN-93G 1/2'"pellet; Bio-Serv) either enriched with $10 \% \mathrm{~L}$-serine $(\mathrm{w} / \mathrm{w})$, enriched with $10 \%$ L-alanine $(\mathrm{w} / \mathrm{w})$, or unsupplemented. Baseline blood plasma samples were taken immediately before mice were placed on enriched diets, and mice were allowed to eat ad libitum. Subsequent blood samples were drawn at 3 -day intervals over a 15-day period. To assess the neurological outcome measures, the L-alanine- and L-serine-enriched diets were administered for up to 12 months, and behavioral and sensory testing occurred at 2-month intervals (see below).

\section{Amino acid analysis}

Amino acids in mouse tissues were analyzed on a Zorbax Eclypse AAA column $(150 \times 4.5 \mathrm{~mm}, 5 \mu \mathrm{M}$; Agilent $)$ according to the manufacturer's instructions. For detection, a Shimadzu LC2010 HPLC system connected to a fluorescence detector (Hewlett Packard) was used. Plasma amino acids were measured using a Biochrom 30 Amino Acid Analyzer with lithium high-performance column and post-column photometric detection with ninhydrin reagent. Samples were deproteinized with buffer containing an internal standard, then centrifuged, after which supernatant was analyzed.

\section{Lipid analysis}

Sphingoid bases in plasma and tissue were analyzed according to a previously described method $(10,13)$, except isotopically labeled SA (d7), SO (d7), and deoxymethyl-SA (d5) were used as internal extraction and run standards (Avanti Polar Lipids).

\section{Tissue and plasma collection}

Blood was collected from the tail veins in lithium heparin-lined capillary tubes. Samples were spun at $2500 \mathrm{~g}$ for 15 minutes. Plasma was collected and then stored at $-80^{\circ} \mathrm{C}$. Mice at 11 and 15 months of age were anesthetized and perfused transcardially with $0.9 \%$ saline. The liver, brain, spinal cord, and sciatic nerves were harvested, flash frozen, and stored at $-80^{\circ} \mathrm{C}$.

\section{Behavioral assays}

Motor performance. Motor function was assessed by rotarod analysis in an accelerating rod paradigm. The mouse was placed on top of the beam, facing away from the experimenter's view, in the orientation opposite to that of its rotation, so that forward locomotion is necessary for fall avoidance. The rotarod accelerated gradually without jerks from 0 to $35 \mathrm{rpm}$ over a 2-minute trial, and latency to fall was recorded manually. Each mouse was given 3 trials with a 15 -minute interval between trials on 5 consecutive days. Mice were tested at 3-5 (L-alanine, 2 months), 13-15 (L-serine, 2 months), and 2-12 (L-serine, 10 months) months of age.

Mechanical and thermal sensitivity (von Frey and hotplate). To assess mechanical allodynia, animals were placed on an elevated wire grid, and the lateral portion of the plantar surface of the hind paw was stimulated using von Frey monofilaments $(0.008-8 \mathrm{~g})$. The withdrawal threshold was determined as the filament at which the animal withdrew its paw at least twice in 10 applications. Thermal hyperalgesia was assessed with a hotplate apparatus (Ugo Basile). Animals were placed on a flat metal surface surrounded by a cylindrical plexiglas barrier to prohibit jumping off and were allowed to acclimatize at ambient temperature to the novel environment. After 10 minutes' rest between acclimatization and trial periods, mice were placed on the plate set at a constant temperature of $50^{\circ} \mathrm{C}$ or $52^{\circ} \mathrm{C}$. Time to the first paw lick was measured by an observer using a foot-controlled timer. To prevent tissue damage to the paws, a maximum cutoff time of 20 seconds was used.

\section{Morphological analysis}

After supplementation, morphological analysis was performed according to methods previously described (13). The mice were perfused with $3 \%$ formaldehyde and $3 \%$ glutaraldehyde. The distal sciatic nerves were 
dissected out and postfixed in $2.5 \%$ glutaraldehyde overnight. The nerves were osmicated with $1 \%$ osmium tetroxide and embedded in an araldite resin. For axon distribution, 10 random nonoverlapping crosssectional regions were imaged at $\times 1,900$ magnification of $2-3$ mice per group (L-serine versus L-alanine). Using Photoshop, tracings of axons and myelin sheaths were filled to create a solid black area representing the cross-sectional surface area of myelin and axons. Using the particle analysis function in Image J, the surface areas of axon and myelin were computed. Axon diameter, total diameter of myelinated axon, myelin thickness, and g ratios (axon diameter/total axon and myelin diameter) were calculated from surface areas. Axon diameters grouped by size were plotted to show relative size distribution; mean and SD were calculated from animal averages.

\section{Sperm analysis}

Before transcardial perfusion, testes and epididymis were removed and placed in phosphate-buffered saline. The caudal epididymis was separated for sperm analysis. Both testes from each animal were separated, weighted in an electronic balance, and fixed with Bouin solution. The sperm suspension was prepared by mincing the caudal epididymis in $1 \mathrm{ml}$ human tubal fluid with $10 \%$ FBS. For analysis, the sperm suspension was in culture for 1 hour at $5 \% \mathrm{CO}_{2}$ in an air incubator. Sperm count was analyzed in a Makler chamber. Motility was assessed by counting the number of motile sperm with forward progression, motile sperm without forward progression, and nonmotile sperm. Fixed testes were embedded in paraffin and stained with hematoxylin and eosin for histology.

\section{Human L-serine supplementation trial}

14 C133Y SPTLC1 mutant carriers were recruited for a 10-week oral L-serine supplementation trial with a monitored 2 -week washout phase after the end of the trial. Subjects received daily supplements of powdered L-serine (mixed in water for consumption) on a low- or high-dose schedule (200 or $400 \mathrm{mg} / \mathrm{kg}$ body weight, respectively; $n=7$ per group). Randomization to high or low dose was stratified by disease severity and gender. Patient characteristics are shown in Supplemental Table 1.

A clinical assessment was carried out before and after the washout phase. We measured dSL levels in the fasting and postprandial states. Thereafter, blood was drawn weekly in the fasting state in the first 4 weeks, and then every second week. Patients were instructed to maintain an otherwise normal diet throughout the study.

1. Bejaoui K, et al. Confirmation of linkage of type 1 hereditary sensory neuropathy to human chromosome 9q22. Neurology. 1999;52(3):510-515.

2. Bejaoui $\mathrm{K}$, et al. SPTLC1 is mutated in hereditary sensory neuropathy, type 1. Nat Genet. 2001; 27(3):261-262.

3. Dawkins JL, Hulme DJ, Brahmbhatt SB, Auer-Grumbach M, Nicholson GA. Mutations in SPTLC1, encoding serine palmitoyltransferase, long chain base subunit-1, cause hereditary sensory neuropathy type I. Nat Genet. 2001;27(3):309-312.

4. Rotthier A, et al. Mutations in the SPTLC2 subunit of serine palmitoyltransferase cause hereditary sensory and autonomic neuropathy type I. Am J Hum Genet. 2010;87(4):513-522.

5. Hanada K. Serine palmitoyltransferase, a key enzyme of sphingolipid metabolism. Biochim Biophys Acta. 2003;1632(1-3):16-30.

6. Houlden $\mathrm{H}$, et al. Clinical, pathological and genetic characterization of hereditary sensory and autonomic neuropathy type 1 (HSAN I). Brain. 2006; 129(pt 2):411-425.

7. Rotthier A, et al. Genes for hereditary sensory and autonomic neuropathies: a genotype-phenotype correlation. Brain. 2009;132(pt 10):2699-2711.

\section{Statistics}

Unless otherwise indicated, all bar graphs are expressed as mean \pm SEM percent of age-matched, untreated WT controls. 1-way ANOVA was used to assess the effects of diet on behavioral (von Frey) and biochemical (dSL accumulation in various tissues) measures. Subsequently, significant findings were further explored by Bonferroni multiple-comparison test. Human and murine biochemical (plasma dSL levels) and murine behavioral (hotplate, rotarod) performance were assessed with repeated-measure ANOVAs. When appropriate, repeated-measure ANOVAs were also conducted to determine the between-subjects effect of duration of treatment (2 versus 10 months) on behavioral performance. Statistical significance was calculated with 2-tailed Student's $t$ tests. Unless otherwise indicated, a $P$ value less than 0.05 was considered significant. Statistical analyses were performed using SPSS.

\section{Study approval}

The animal protocol was reviewed and approved by the Massachusetts General Hospital Subcommittee on Research Animal Care (protocol no. 2003N000270; Charlestown, Massachusetts, USA). Approval to perform this study in humans was obtained from the IRB at Massachusetts General Hospital (protocol no. 2009P000341; Boston, Massachusetts, USA). All participants provided written informed consent before engaging in any study-related activities.

\section{Acknowledgments}

We thank Cotton Widdicombe and Martin Selig for their assistance in the rodent studies and Ourania Giannikopoulos for her assistance in the human studies. The work was supported by the NIH (grants K08NS52550 and R01 NS072446) and the Deater Foundation (to F.S. Eichler); by the European Union (grant LSHM-CT2006-037631; to T. Hornemann and A. von Eckardstein); and by the Zurich Center for Integrative Human Physiology (ZIHP) and the Gebert-Rüf Foundation (grant GRS-047/09; to T. Hornemann).

Received for publication February 11, 2011, and accepted in revised form September 7, 2011.

Address correspondence to: Florian S. Eichler, Massachusetts General Hospital, Department of Neurology, 55 Fruit Street, ACC 708, Boston, Massachusetts 02114, USA. Phone: 617.724.7121; Fax: 617.643.6374; E-mail: feichler@partners.org.
8. Hornemann T, et al. A systematic comparison of all mutations in hereditary sensory neuropathy type I (HSAN I) reveals that the G387A mutation is not disease associated. Neurogenetics. 2009; 10(2):135-143.

9. Dedov VN, Dedova IV, Merrill AH Jr, Nicholson GA. Activity of partially inhibited serine palmitoyltransferase is sufficient for normal sphingolipid metabolism and viability of HSN1 patient cells. Biochim Biophys Acta. 2004;1688(2):168-175.

10. Penno A, et al. Hereditary sensory neuropathy type 1 is caused by the accumulation of two neurotoxic sphingolipids. J Biol Chem. 2010;285(15):11178-11187.

11. Gable K, Gupta SD, Han G, Niranjanakumari S, Harmon JM, Dunn TM. A disease-causing mutation in the active site of serine palmitoyltransferase causes catalytic promiscuity. J Biol Chem. 2010;285(30):22846-22852.

12. Zitomer NC, et al. Ceramide synthase inhibition by fumonisin B1 causes accumulation of 1deoxysphinganine: a novel category of bioactive 1-deoxysphingoid bases and 1-deoxydihydroceramides biosynthesized by mammalian cell lines and animals. J Biol Chem. 2009;284(8):4786-4795.

13. Eichler FS, et al. Overexpression of the wild-type
SPT1 subunit lowers desoxysphingolipid levels and rescues the phenotype of HSAN1. J Neurosci. 2009;29(46):14646-14651.

14. Denny-Brown D. Hereditary sensory radicular neuropathy. J Neurol Neurosurg Psychiatry. 1951; 14(4):237-252.

15. Lindahl AJ, Lhatoo SD, Campbell MJ, Nicholson G, Love S. Late-onset hereditary sensory neuropathy type I due to SPTLC1 mutation: autopsy findings. Clin Neurol Neurosurg. 2006;108(8):780-783.

16. Reimann HA, McKechnie WG, Stanisavljevic S. Hereditary sensory radicular neuropathy and other defects in a large family: reinvestigation after twenty years and report of a necropsy. Am J Med. 1958; 25(4):573-579.

17. McCampbell A, et al. Mutant SPTLC1 dominantly inhibits serine palmitoyltransferase activity in vivo and confers an age-dependent neuropathy. Hum Mol Genet. 2005;14(22):3507-3521.

18. Hirabayashi Y, Furuya S. Roles of l-serine and sphingolipid synthesis in brain development and neuronal survival. Prog Lipid Res. 2008;47(3):188-203.

19. de Koning TJ. Treatment with amino acids in serine deficiency disorders. J Inherit Metab Dis. 2006; 29(2-3):347-351. 
20. de Koning TJ, Snell K, Duran M, Berger R, Poll-The BT, Surtees R. L-serine in disease and development. Biochem J. 2003;371(pt 3):653-661.

21. Bejaoui K, et al. Hereditary sensory neuropathy type 1 mutations confer dominant negative effects on serine palmitoyltransferase, critical for sphingolipid synthesis. J Clin Invest. 2002; 110(9):1301-1308.

22. Gable K, et al. Mutations in the yeast LCB1 and LCB2 genes, including those corresponding to the hereditary sensory neuropathy type I mutations, dominantly inactivate serine palmitoyltransferase. J Biol Chem. 2002;277(12):10194-10200.

23. Hojjati MR, Li Z, Jiang XC. Serine palmitoyl-CoA transferase (SPT) deficiency and sphingolipid levels in mice. Biochim Biophys Acta. 2005;1737(1):44-51.

24. Humpf HU, et al. Acylation of naturally occurring and synthetic 1-deoxysphinganines by ceramide synthase. Formation of N-palmitoyl-aminopentol produces a toxic metabolite of hydrolyzed fumonisin, AP1, and a new category of ceramide synthase inhibitor. J Biol Chem. 1998;273(30):19060-19064.

25. Salcedo M, et al. The marine sphingolipid-derived compound ES 285 triggers an atypical cell death pathway. Apoptosis. 2007;12(2):395-409.

26. Cuadros R, Montejo de Garcini E, Wandosell F, Faircloth G, Fernandez-Sousa JM, Avila J. The marine compound spisulosine, an inhibitor of cell proliferation, promotes the disassembly of actin stress fibers. Cancer Lett. 2000;152(1):23-29.

27. Sandhoff R, et al. Novel class of glycosphingolipids involved in male fertility. J Biol Chem. 2005; 280(29):27310-27318.

28. Takamiya K, et al. Complex gangliosides are essential in spermatogenesis of mice: possible roles in the transport of testosterone. Proc Natl Acad Sci U S A. 1998;95(21):12147-12152.

29. Bertea M, et al. Deoxysphingoid bases as plasma markers in Diabetes mellitus. Lipids Health Dis. 2010;9:84.

30. Said G. Diabetic neuropathy-a review. Nat Clin Pract Neurol. 2007;3(6):331-340. 